\title{
Appraisal of One Health approach amid COVID-19 and zoonotic pandemics: insights for policy decision
}

\author{
Muhammad Imran Arshad ${ }^{1}$ (D) - Hilal Ahmad Khan ${ }^{1} \cdot$ Bilal Aslam $^{2} \cdot$ Junaid Ali Khan ${ }^{3}$
}

Received: 30 June 2020 / Accepted: 11 November 2020 / Published online: 18 November 2020

(C) Springer Nature B.V. 2020

The coronavirus disease 2019 (COVID-19) pandemic was declared as a public health emergency of international concern, and it caused huge economic and health losses globally. Facing a pandemic has called into question the preparedness and approaches taken to control, contain, and quarantine all over the world. Bordering with two hot-spots (i.e., China and Iran) of COVID-19 and representing a human population of 220 million, a havoc public health situation of COVID-19 is prevailing in Pakistan (WHO, Badshah et al. 2020). Currently, there are 209,013 confirmed cases of COVID-19 and 4304 deaths in Pakistan with top in surge of daily cases in the South Asia. The global public health emergency of COVID19 associated with 2019-nCoV/SARS-CoV-2 virus necessitates the action plan to be implemented at the animalhuman-environment niches of this pandemic (Jiang et al., 2020). The basic reproduction number (R0) of disease transmissibility indicator for 2019-nCoV/SARS-CoV-2 ranges from 3 to 4 , and it is regionally variable. It is plausibly needed to initiate an integrated strategic framework of collaboration and capacity building to cope with alarming situation of COVID-19.

In today's highly interconnected world, the people, animals, vectors, international travel, population density, and environment play an important role in the spread or transmission of diseases such as COVID-19. More than 70\% of emerging infectious diseases (EIDs) and six out of ten infectious diseases of human are linked to animals, and such transmission is

Muhammad Imran Arshad drimranarshad@yahoo.com

1 Institute of Microbiology, University of Agriculture Faisalabad, Faisalabad 38040, Pakistan

2 Department of Microbiology, Government College University Faisalabad, Faisalabad, Pakistan

3 Institute of Physiology \& Pharmacology, University of Agriculture, Faisalabad, Pakistan controlled by several environmental factors (Mwangi et al., 2016). Most of high-profile diseases or pathogens such as highly pathogenic influenza, SARS, MERS-CoV, Nipah, Hendra, Dengue, Ebola, and Zika viruses emerged from developing and least developed countries heed a crossdisciplinary approach to mitigate pathogens. The recent pandemic of COVID-19, probably of zoonotic origin, necessitates appraisal of multi-disciplinary "One Health" approach by coordinating human, animal, and environment professionals (Zhou et al., 2020; Andersen et al., 2020). Community health is a complex multi-factorial process, and many components are covered under the umbrella of One Health. The One Health core competencies (collaboration, coordination, partnership, leadership, communication, ethics, systems thinking, and management) have been identified in responding pandemics or public health issues globally such as Bellagio working group, USAID/RESPOND, SMM, Rome Synthesis, and PREDICT (Frankson et al., 2016).

The havoc situation of COVID-19 has led to mobilization of preparedness and action plans to prevent and control at global level. In other words, how to "flatten the COVID-19 curve" has been central to the debate and plans of action. Integrated disease surveillance, prophylaxis, infection prevention, and control strategies are plausibly needed to circumvent global spread of 2019-nCoV/SARS-CoV-2. The global COVID-19 issue is quintessentially associated with One Health paradigm; therefore, adaptability of One Health approach is imperative to mitigate spread, spillover events, prevalence, and persistence of 2019-nCoV/SARS-CoV-2 at the animal-human-environment interface. The host (age, sex, immune status, genetic factors, traveling, behavior), agent (virulence, infectiousness, pathogenecity, spillover), and environmental (hygiene, fomites, persistence, distancing) determinants are crucially involved in transmission dynamics of COVID-19. Breaking the above chain of infection and disease variables could be effective strategy to dampen surge of COVID-19. Control of animal reservoirs (i.e., bats, canine, pangolin, dogs, snakes, and wild birds) of 2019-nCoV/ 
SARS-CoV-2 would be needed to halt the panic situation and re-emergence of this menace globally.

Empiric and empathic approaches should be adopted to contain droplet, aerosol, and human-to-human infections from suspected COVID-19 patients. Administrative, environmental, and engineering controls are dispensable to prevent spread of COVID-19 in the community. Strict compliance with principles of water and sanitation for health (WASH) and respiratory hygiene or cough etiquette may significantly dampen the spread of COVID-19 viruses and other contagious respiratory diseases. Concomitance to One Health approach, the WHO has introduced eight pillars (i.e., coordination-monitoring, risk communication/community engagement, surveillanceinvestigation-rapid diagnosis, control points of entry, case management, infection prevention and control, operational logistic support, and national laboratory capacity enhancement) of preparedness and response plan for countries to cope with COVID-19 (WHO emergency guidelines). These pillars and One Health core competencies might be followed for effective control of COVID-19 along with real time surveillance, infection control, and preventive strategies. In developing and vulnerable countries like Pakistan, training of personal protective equipment (PPE), biosafety, isolation and quarantine (I\&Q), basic laboratory disease diagnostic, biosurveillance, and awareness of public or relevant personnel via outreach are direly needed (Stroud et al., 2016; Karamouzian \& Madani, 2020).

Finally, an integrated community response, global collaboration, holistic approaches, strict compliance with infection control, and relevant training of professionals in One Health would be needed for sustainable global health security. It provides insights for policy making to implement disease risk assessment, preparedness, and response plans for COVID19. Defining of One Health priority agenda, promotion of One Health research, policy, education, and advocacy with political commitment are crucially needed for healthy wellbeing and economic growth regionally or globally. Hence, recognizing inextricable animal-human-environment link, development of technical One Health workforce, mitigation of occupational risk, awareness, and vaccination strategies are needed at policy making, stakeholder, and ministry levels to better prepare for epidemics and zoonotic pandemics.

Acknowledgements The authors thank US National Academy of Sciences (US-NAS) and Pakistan Academy of Sciences (PAS) for One Health Fellowship initiative in Pakistan. Muhammad Imran Arshad is One Health Fellow of US-NAS and Member of PAS.

Authors' contributions Muhammad Imran Arshad: conceptualization, data curation, writing-original draft. Hilal Ahmad Khan: conceptualization, writing - review and editing. Bilal Aslam: conceptualization, writing - review and editing. Junaid Ali Khan: proof-reading and editing.

\section{Compliance with ethical standards}

Conflict of interest The authors declare that they have no conflict of interests.

\section{References}

Andersen, K. G., Rambaut, A., Lipkin, W. I., Holmes, E. C., \& Garry, R. F. (2020). The proximal origin of SARS-CoV-2. Nature Medicine, 26(4): 450-452. doi: https://doi.org/10.1038/s41591-020-0820-9

Badshah, S. L., Ullah, A., Badshah, S. H., \& Ahmad, I. (2020). Spread of Novel Coronavirus by Returning Pilgrims from Iran to Pakistan. Journal of Travel Medicine, 27(3): taaa044. doi: https://doi.org/10. 1093/jtm/taaa044

Frankson, R., Hueston, W., Christian, K., Olson, D., Lee, M., Valeri, L., Hyatt, R., Annelli, J., \& Rubin, C. (2016). One Health Core Competency Domains. Frontiers in Public Health, 4: 192. eCollection 2016. doi: https://doi.org/10.3389/fpubh.2016.00192.

Jiang, S., Shi, Z., Shu, Y., Song, J., Gao, G. F., Tan, W., \& Guo, D. (2020). A distinct name is needed for the new coronavirus. Lancet, 395(10228): 949. doi: https://doi.org/10.1016/S0140-6736(20) 30419-0

Karamouzian, M., \& Madani, N. (2020). COVID-19 Response in the Middle East and North Africa: Challenges and Paths Forward. Lancet Global Health, S2214-109X(20)30233-3. doi: https://doi. org/10.1016/S2214-109X(20)30233-3

Mwangi, W., Figueiredo, P. de., \& Criscitiello, M. F. (2016). One Health: Addressing global challenges at the nexus of human, animal, and environmental health. PLoS Pathogens, 12(9): e1005731. doi: https://doi.org/10.1371/journal.ppat.1005731

Stroud, C., Kaplan, B., Logan, J. E., \& Gray, G. C. (2016). One Health training, research, and outreach in North America. Infection Ecology and Epidemiology, 6: 33680. doi: https://doi.org/10.3402/ iee.v6.33680

Zhou, P., Yang, X. L., Wang, X. G., Hu, B., Zhang, L., Zhang, W., Si, H. R., Zhu, Y., Li, B., Huang, C. L., Chen, H. D., Chen, J., Luo, Y., Guo, H., Jiang, R. D., Liu, M. Q., Chen, Y., Shen, X. R., Wang, X., Zheng, X. S., Zhao, K., Chen, Q. J., Deng, F., Liu, L. L., Yan, B., Zhan, F. X., Wang, Y. Y., Xiao, G. F., \& Shi, Z. L. (2020). A pneumonia outbreak associated with a new coronavirus of probable bat origin. Nature, 579(7798): 270-273. doi: https://doi.org/10.1038/ s41586-020-2012-7

\section{Further Reading}

https://covid19.who.int/; http://covid.gov.pk/stats/pakistan (accessed Jun 30, 2020)

https://www.who.int/emergencies/diseases/novel-coronavirus-2019/technical-guidance/early-investigations (accessed Jun 20 2020)

Publisher's note Springer Nature remains neutral with regard to jurisdictional claims in published maps and institutional affiliations. 